\author{
EVS25 \\ Shenzhen, China, Nov5-9, 2010
}

\title{
Impact of cut edges on magnetization curves and iron losses in e-machines for automotive traction
}

\author{
Lode Vandenbossche ${ }^{1}$, Sigrid Jacobs ${ }^{2}$, François Henrotte ${ }^{3}$ and Kay Hameyer ${ }^{3}$ \\ ${ }^{I}$ ArcelorMittal Global R\&D Gent, J.F. Kennedylaan 3, BE-9060 Zelzate, Belgium \\ ${ }_{2}^{2}$ (presenting author) ArcelorMittal Global R\&D, J.F. Kennedylaan 51, BE-9042 Gent, Belgium \\ E-mail: sigrid.jacobs@arcelormittal.com \\ ${ }^{3}$ IEM RWTH-Aachen University, Schinkelstrasse 4, DE-52056 Aachen, Germany
}

\begin{abstract}
At last year's edition of EVS, we presented an improved model for iron losses prediction in Permanent Magnet Synchronous Machines (PMSM) [1]. The benefit of this model holds in that it fits more closely the real material behavior than the standard Steinmetz or Bertotti approaches [2], by including 1) magnetic material characteristics measured at high frequency and 2) an improved representation of iron losses at the approach to saturation (by introducing a higher order term in $\mathrm{J}$ ). We are taking this model a step further by considering now another phenomenon impacting iron losses in electrical machines: the decrease of magnetic permeability and the increase of local hysteresis loss at the vicinity of lamination edges due to the cutting process. This paper presents a quantitative analysis of the impact of lamination processing (cutting, punching, etc) for high quality low loss electrical steels used in automotive traction applications. It is important to perform the analysis over a wide frequency range, because of the large speed range of PMSM drives in automotive applications and the presence of higher harmonics (PWM supply). Our approach consists in measuring the material characteristics for sample sets with different ratios of degraded vs. non degraded material and at various frequencies. Starting from that experimental data we propose a method to determine the local magnetization curves, as function of distance from the cut edge. These local material characteristics can then be implemented in a FE model so that the effect of punching on the machine performance can be determined quantitatively: (1) the cutting impact on magnetization modification allows more precise field calculations; (2) a proposition is made on the implementation of the cutting impact on the loss calculations in post processing via an enhanced version of the loss model developed in
\end{abstract} [1].

Keywords-Material models for finite element computation, iron losses, magnetization curve, low loss fully processed electrical steels, machine design optimization

\section{Introduction}

The industrial processing of steel laminations (cutting, punching, etc.) causes a significant degradation of its magnetic material properties. Locally near the cutting edge the microstructure of the steel (dislocations, internal stresses, grain morphology) is affected by the mechanical cutting or punching, which results in a decrease of magnetic permeability and an increase of hysteresis losses in the region near the cutting edge [3-9]. ArcelorMittal quantified this effect in the past for typical electrical steel grades used in industry machines $(50 \mathrm{~Hz}$ applications) [4,5]. In 
this paper, we present a new study with focus on the electrical steel grades used for automotive traction machines and their specific application range (for higher frequencies than $50 \mathrm{~Hz}$ ).

\section{Experimental procedure}

\subsection{Sample preparation}

A number of samples of $80 \mathrm{~mm}$ by $260 \mathrm{~mm}$ are cut by electrical discharge wire cutting (spark erosion). The outer edges of these $80 \mathrm{~mm}$ wide samples are assumed to be unaffected by this cutting technique [4-5]. The length direction of the samples corresponds to the rolling direction of the sheet. In this paper, the material under test is a fully processed electrical steel grade M235-35A.

Further, some of these samples are cut in smaller strips by guillotine cutting (cutting blade makes a small angle with the plane of the sheet), which simulates the actual punching used in the industrial process. This results in four different sample sets, one with $\mathrm{N}=6$ additional guillotine cut edges, one with $\mathrm{N}=14$, one with $\mathrm{N}=26$ and one with $\mathrm{N}=42$ cut edges. The total width remains $80 \mathrm{~mm}$. In more detail, the sample set with $\mathrm{N}=6$ consists of 4 strips of $20 \mathrm{~mm}$ in width, the sample set with $\mathrm{N}=14$ consists of 8 samples of $10 \mathrm{~mm}$ in width, and so on: 14 strips of 5.71 $\mathrm{mm}$ and 22 strips of $3.64 \mathrm{~mm}$. A sample of 80 $\mathrm{mm}$ width without additional cut edges is taken as the reference sample.

\subsection{Magnetic measurements}

In order to characterize the cut edge effect on both the single valued (virgin) magnetization curve $\mathrm{J}(\mathrm{H})$ and on the losses at elevated frequencies (from $50 \mathrm{~Hz}$ up to $1 \mathrm{kHz}$ ), magnetic measurements are performed on these five sets of samples of M235-35A (always $80 \mathrm{~mm}$ width in total, but with different number $\mathrm{N}$ of guillotine cut edges), with a single sheet tester having a magnetic path length of $226 \mathrm{~mm}$.

Due to the cutting, a spatial distribution of magnetic polarization $\mathrm{J}(\mathrm{x})$ as function of the distance $\mathrm{x}$ from the cutting edge is present. Hence, in the context of this paper, it's important to emphasize the following inherent aspect of magnetic measurements: the measured $\mathrm{J}$ at a certain time instant $\mathrm{t}$ is actually a mean value, averaged out in space, over the cross section of the sample under test. In the following, this is highlighted by denoting the measured magnetic polarization as $\mathrm{J}_{\text {avg }}(\mathrm{t})$.

When performing the magnetic measurements, the amplitude value of the magnetic polarization $\hat{\mathrm{J}}_{\mathrm{avg}}$ (averaged out in space) is controlled, and also the waveform of one cycle of the induced voltage $\mathrm{V}_{\text {sec }}(\mathrm{t}) \sim-\mathrm{dJ}_{\mathrm{avg}}(\mathrm{t}) / \mathrm{dt}$ is controlled to be sinusoidal. Measurements are performed for different polarization amplitudes $\hat{\mathrm{J}}_{\text {avg }}$, and for the following excitation frequencies: $50,200,400,700 \mathrm{~Hz}$ and 1 $\mathrm{kHz}$. Peak (amplitude) values of $\mathrm{H}(\mathrm{t})$ and $\mathrm{J}_{\mathrm{avg}}(\mathrm{t})$ are recorded (denoted by $\hat{H}$ and $\hat{\mathrm{J}}_{\mathrm{avg}}$ ), and also the losses $\mathrm{P}$, in $\mathrm{W} / \mathrm{kg}$, are retrieved.

\subsection{Results regarding the virgin magnetic polarization curve}

Fig. 1 shows the change in shape of the virgin magnetic polarization curve. The strongest effect is noted in the lower $\mathrm{H}$ and $\mathrm{J}$ area. Fig. 2 and 3 show the relative permeability $\mu \mathrm{r}$, being the amplitude permeability $\mu_{\mathrm{r}}=\hat{\mathrm{J}}_{\mathrm{avg}} /(\mu 0 \hat{\mathrm{H}})$. When the number of cut edges is increased, the permeability below $1 \mathrm{kA} / \mathrm{m}$ (or $1.5 \mathrm{~T}$ ) reduces drastically. Clearly the impact of cutting is strongest when magnetization is realized via magnetic domain boundary displacement. Above the knee-point, when rotational magnetization is the predominant phenomenon, the dislocations created during the punching hinder the magnetization process less.

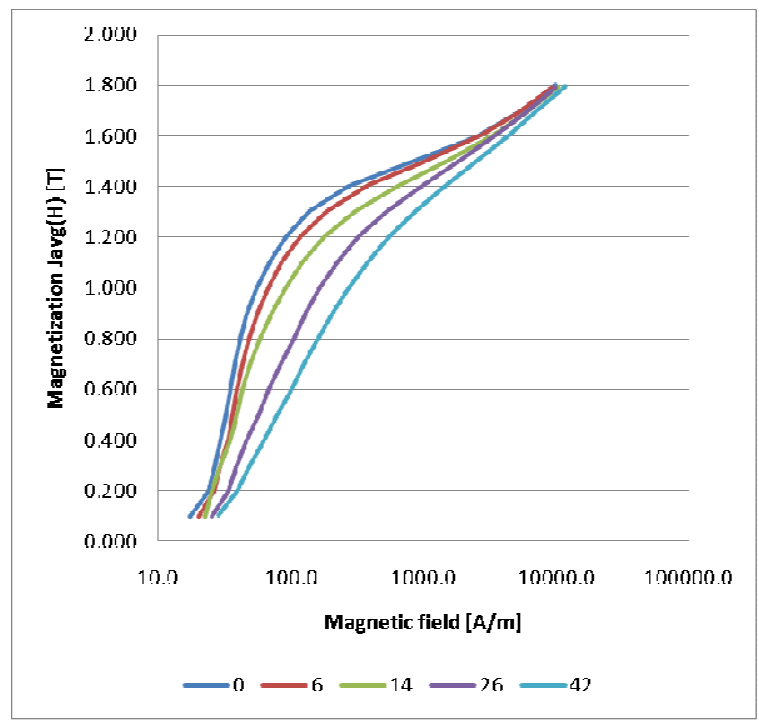

Figure 1: $\mathrm{J}_{\text {avg }}(\mathrm{H})$ characteristics for several values of the number of cutting edges $\mathrm{N}$. 


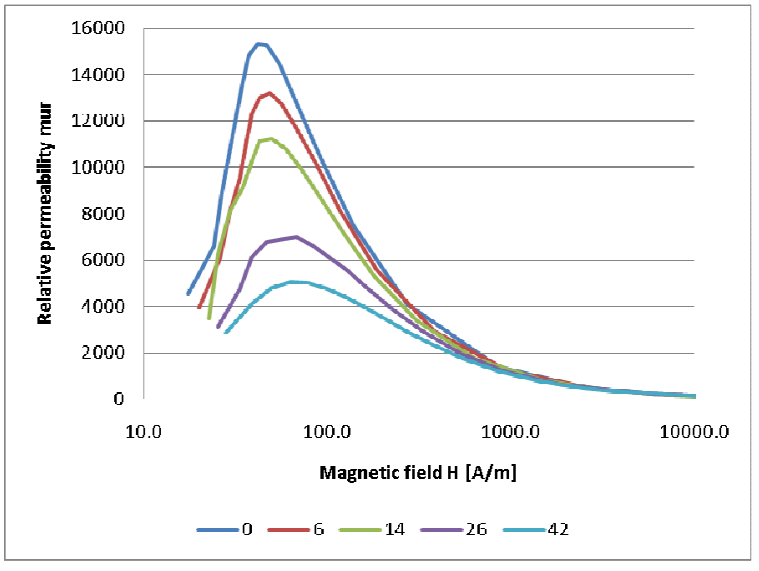

Figure 2: $\mu_{\mathrm{r}}(\mathrm{H})$ characteristics for several values of the number of cutting edges $\mathrm{N}$.

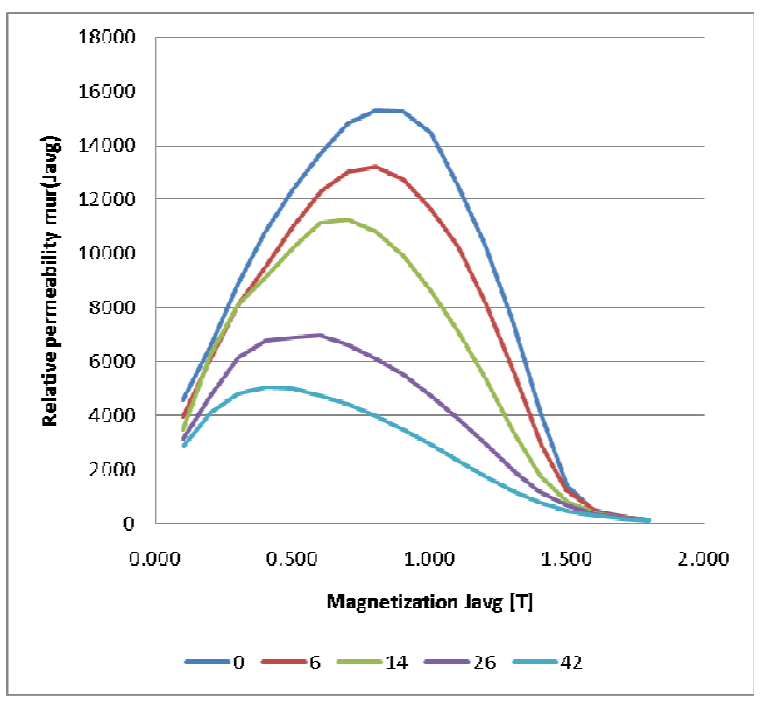

Figure 3: $\mu_{\mathrm{r}}\left(\hat{\mathrm{J}}_{\mathrm{avg}}\right)$ characteristics for several values of the number of cutting edges $\mathrm{N}$.

\subsection{Results regarding the magnetization loops}

Fig. 4 to 6 show the magnetization loops at the polarization levels of $1 \mathrm{~T}$ and $1,5 \mathrm{~T}$, resp. at $50 \mathrm{~Hz}$, $400 \mathrm{~Hz}$ and $1 \mathrm{kHz}$. Clearly the strongest impact of cutting is that it is making the loops less squared and more S-shaped, a behavior we already saw in fig. 1. Additionally in these figures, considered for a certain frequency, the widening of the loops due to the cutting can be noted, hence an increase in the coercive fields.

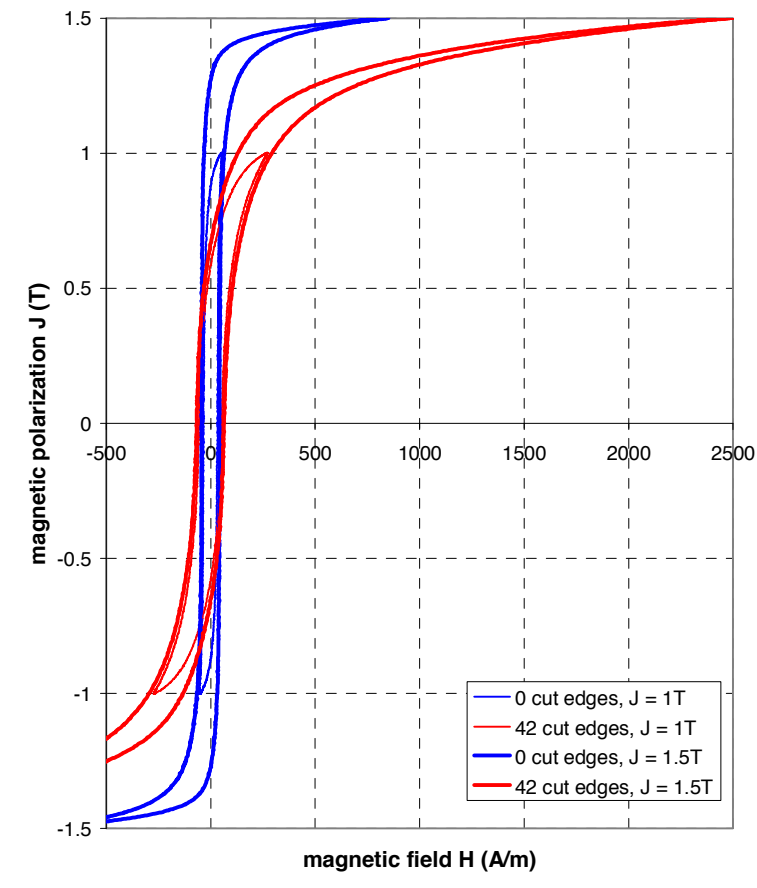

Fig. 4: measured magnetisation loops at $50 \mathrm{~Hz}$

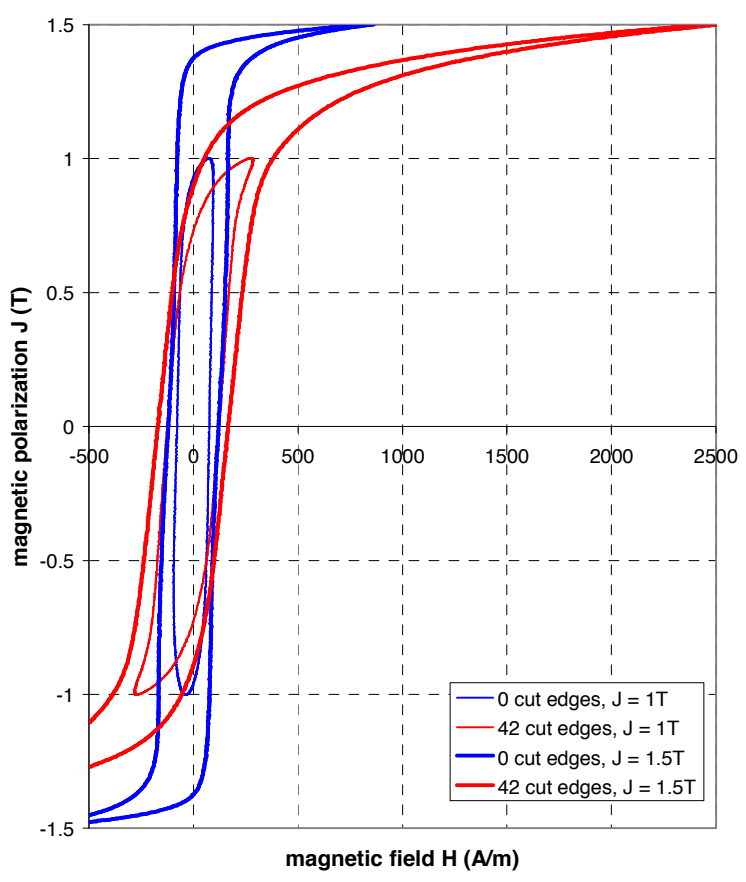

Fig. 5: measured magnetisation loops at $400 \mathrm{~Hz}$ 


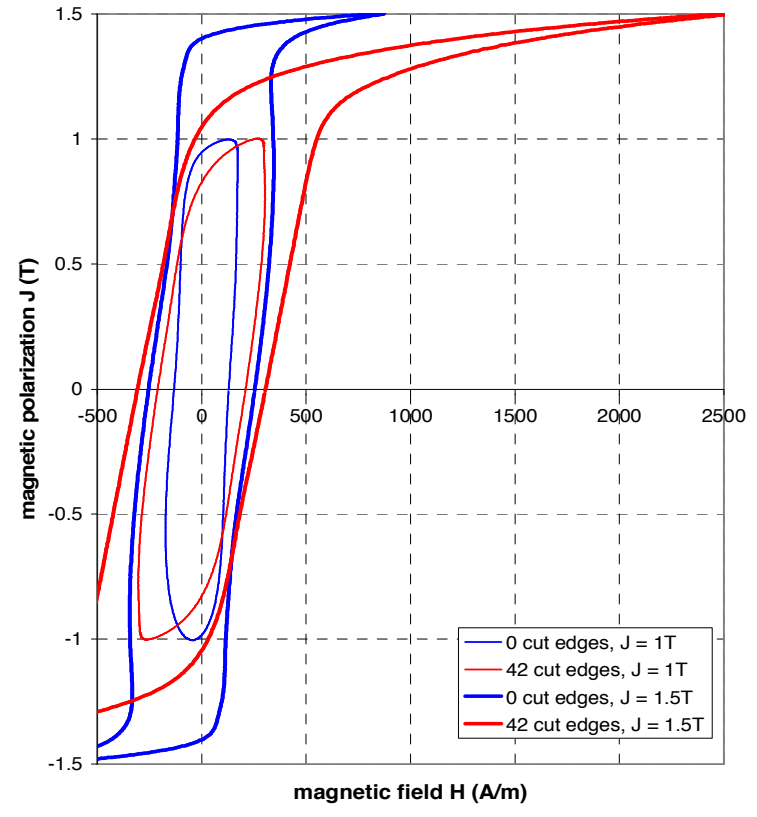

Fig. 6: measured magnetisation loops at $1 \mathrm{kHz}$

Considering the frequency dependence of the loop shape and the associated losses, we can assume that the electrical conductivity (or the chemical composition) is locally not being changed by the cutting process. The (classical Foucault) eddy currents are thus not directly influenced by the cutting technique. On the other hand, the eddy current distribution will (indirectly) be influenced by the cut edge quality, in a sense that a change in the eddy current distribution is a consequence of the variation of the local magnetic flux density $\mathrm{J}(\mathrm{x})$, see section 4.1 .

\subsection{Results regarding the losses}

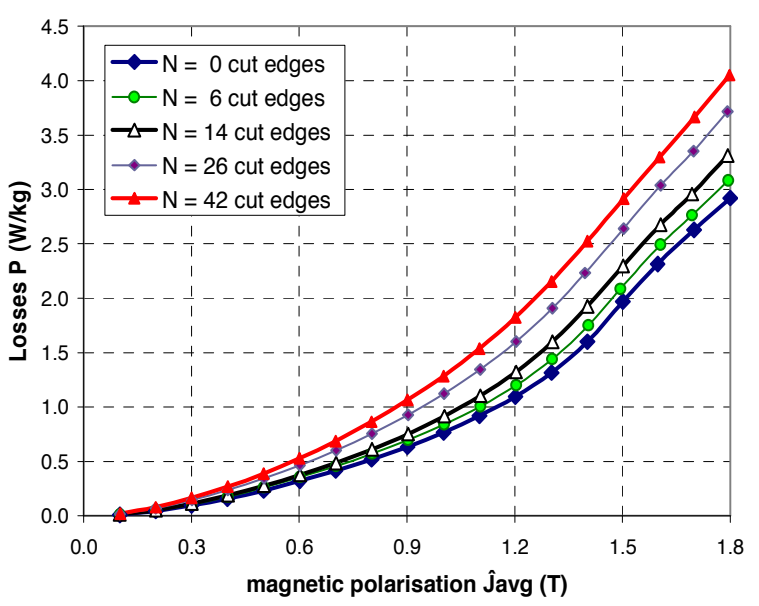

Figure 7: $\mathrm{P}\left(\hat{\mathrm{J}}_{\text {avg }}\right)$ characteristics at $50 \mathrm{~Hz}$, for several values of the number of cutting edges $\mathrm{N}$.

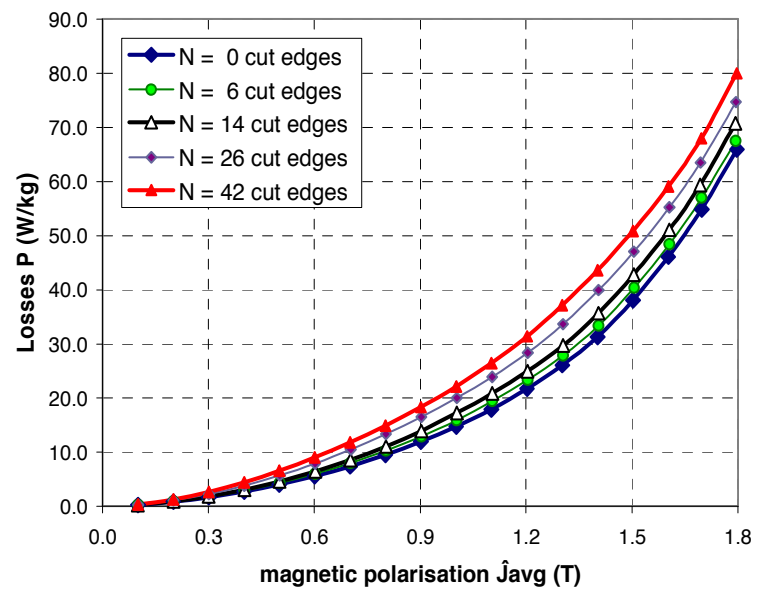

Figure 8: $\mathrm{P}\left(\hat{\mathrm{J}}_{\mathrm{avg}}\right)$ characteristics at $400 \mathrm{~Hz}$, for several values of the number of cutting edges $\mathrm{N}$.

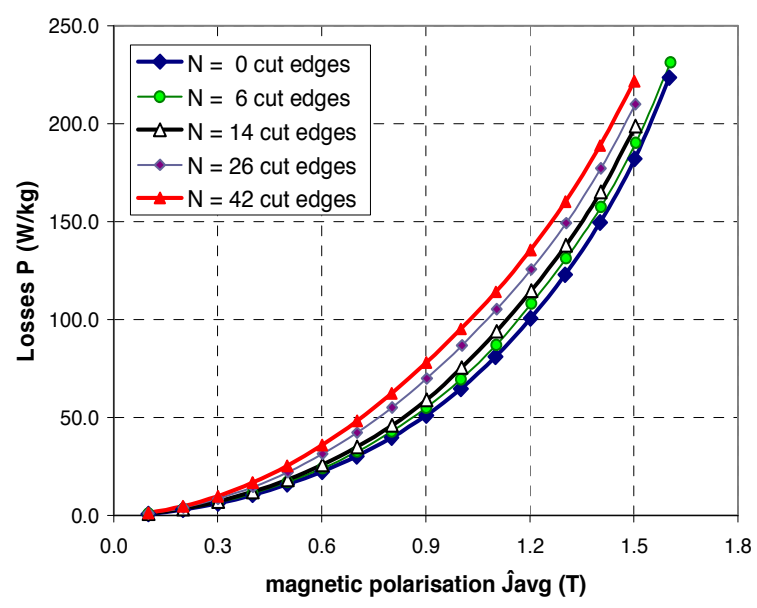

Figure 9: $\mathrm{P}\left(\hat{\mathrm{J}}_{\mathrm{avg}}\right)$ characteristics at $1 \mathrm{kHz}$, for several values of the number of cutting edges $\mathrm{N}$.

Fig. 7 to 9 show the increase in losses $\mathrm{P}\left(\hat{\mathrm{J}}_{\mathrm{avg}}\right)$ with the number of cut edges at $50 \mathrm{~Hz}, 400 \mathrm{~Hz}$ and 1 $\mathrm{kHz}$, respectively. In table 1 the increase of losses with the number of cuts and the frequency is quantified.

Note that these values are obtained with single sheet measurements on samples parallel to rolling direction, whereas standard Epstein measurements are performed with a different magnetic circuit (different magnetic path length definition) and moreover on a mixed sample set (half of the samples are along rolling direction, half is transverse direction). Hence, the results shown in this paper should be interpreted relatively, i.e. in comparison to one another to study relative 
differences, as given by table 2 . The absolute values should be interpreted with care.

Table 1: absolute values of the losses in $\mathrm{W} / \mathrm{kg}$ for three different excitation frequencies, three different sample sets $(\mathrm{N}=0,14$ and 42$)$, and two amplitude values for magnetic polarisation

\begin{tabular}{|c|c|c|c|}
\hline & \multicolumn{3}{|c|}{ Losses $(\mathrm{W} / \mathrm{kg})$ at $\mathbf{J}_{\text {avg }}=1$ T } \\
\hline & reference & 14 cut edges & 42 cut edges \\
\hline $\mathrm{f}=50 \mathrm{~Hz}$ & 0.77 & 0.92 & 1.29 \\
\hline $\mathrm{f}=400 \mathrm{~Hz}$ & 14.69 & 17.24 & 22.15 \\
\hline $\mathrm{f}=1 \mathrm{kHz}$ & 64.81 & 75.63 & 95.24 \\
\hline
\end{tabular}

\begin{tabular}{|c|c|c|c|}
\hline & \multicolumn{3}{|c|}{ Losses $(\mathrm{W} / \mathrm{kg})$ at $\mathbf{J}_{\text {avg }}=1.5 T$} \\
\hline & reference & 14 cut edges & 42 cut edges \\
\hline $\mathrm{f}=50 \mathrm{~Hz}$ & 1.97 & 2.30 & 2.92 \\
\hline $\mathrm{f}=400 \mathrm{~Hz}$ & 38.05 & 42.83 & 50.84 \\
\hline $\mathrm{f}=1 \mathrm{kHz}$ & 182.05 & 198.90 & 221.82 \\
\hline
\end{tabular}

Table 2: relative values of the losses in $\mathrm{W} / \mathrm{kg}$ compared to the reference case $(\mathrm{N}=0)$, for two different sample sets $(\mathrm{N}=14$ and 42$)$, three different excitation frequencies, and two amplitude values of magnetic polarization.

\begin{tabular}{|c|c|c|}
\hline & \multicolumn{2}{|c|}{$\begin{array}{c}\text { Increase of losses at } \hat{\mathbf{J}}_{\text {avg }}=\mathbf{1 T} \\
\text { (compared to reference, } \mathbf{N}=\mathbf{0})\end{array}$} \\
\hline & 14 cut edges & 42 cut edges \\
\hline $\mathrm{f}=50 \mathrm{~Hz}$ & $19.4 \%$ & $67.7 \%$ \\
\hline $\mathrm{f}=400 \mathrm{~Hz}$ & $17.3 \%$ & $50.7 \%$ \\
\hline $\mathrm{f}=1 \mathrm{kHz}$ & $16.7 \%$ & $47.0 \%$ \\
\hline
\end{tabular}

\begin{tabular}{|c|c|c|}
\hline & \multicolumn{2}{|c|}{$\begin{array}{c}\text { Increase of losses at } \hat{\mathbf{J}}_{\text {avg }}=\mathbf{1 . 5 T} \\
\text { (compared to reference, } \mathbf{N}=\mathbf{0} \text { ) }\end{array}$} \\
\hline & 14 cut edges & 42 cut edges \\
\hline $\mathrm{f}=50 \mathrm{~Hz}$ & $16.6 \%$ & $48.0 \%$ \\
\hline $\mathrm{f}=400 \mathrm{~Hz}$ & $12.6 \%$ & $33.6 \%$ \\
\hline $\mathrm{f}=1 \mathrm{kHz}$ & $9.3 \%$ & $21.8 \%$ \\
\hline
\end{tabular}

When comparing in table 2 the losses at same $\hat{\mathrm{J}}_{\text {avg }}$ (e.g. at $1.5 \mathrm{~T}$ ) for the sample set without cuts and the sample with the highest number of cutting edges $(\mathrm{N}=42)$, we see that $\mathrm{P}$ at $1.5 \mathrm{~T} / 50 \mathrm{~Hz}$ increases with $48 \%$, whereas $\mathrm{P}$ at $1.5 \mathrm{~T} / 1 \mathrm{kHz}$ increases still with $22 \%$. The percentage value of this increase of depends on the $\hat{J}_{\text {avg }}$-value at which the comparison is performed.
It is clear that the degradation of the magnetization curve $J_{\text {avg }}(H)$ due to the cutting plays an important role: to obtain the same $\mathrm{J}_{\mathrm{avg}}$, for the sample with $\mathrm{N}=$ 42 cut edges we need to apply more $\mathrm{H}$, resulting in higher losses.

The effect of degraded properties at the cut edge results in a non-uniform distribution of flux over the width of the material. This is also the case during the measurements of the losses: the measured $\mathbf{J}_{\mathrm{avg}}$ has to be envisaged as the value averaged in space (in the bulk, $\mathrm{J}(\mathrm{x})$ will be somewhat higher than $\mathrm{J}_{\mathrm{avg}}$, whereas at the edge, $\mathrm{J}(\mathrm{x})$ decreases).

\section{Mathematic description of the effect of cutting on the $J(H, x)$ curve}

\subsection{Methodology}

\subsubsection{The magnetic permeability decreases locally}

As described in $\S 1$, the $\mathrm{J}$ field locally decreases in the vicinity of a cut edge compared to the value at the bulk of the sheet. Micro-magnetic measurements reported in [3] show that the spatial distribution of $\mathrm{J}(\mathrm{x})$, where $\mathrm{x}$ represents the distance from the edge ( $x=0$ is the edge itself), is parabolic and can have a depth up to $10 \mathrm{~mm}[3,6]$, depending on the actual definition of this depth.

Ampere's law imposes the magnetic field $\mathrm{H}$ to be uniform over the sample when performing the SST measurements. Therefore, the effect of cutting the lamination can be seen as a local inhomogeneous decrease of the magnetic permeability $\mu$. We note this variation of the permeability $\Delta \mu(\mathrm{H}, \mathrm{x})$.

\subsubsection{Maximal permeability drop $\Delta \mu_{\text {cut }}(\mathrm{H})=\Delta \mu(\mathrm{H}, \mathrm{x}=0)$ is a material constant}

The density of additional dislocations and the residual stresses induced by cutting is maximal at the cut edge, and decreases as one enters the lamination. This maximum density is linked to the plastic strain attained by the material during the cutting process, which is itself linked to the applied stress. In case of cutting, this applied stress must have reached the rupture stress, which is a material constant. One can thus assume that the increase of the dislocation density at the cut edge, and hence $\Delta \mu_{\text {cut }}(\mathrm{H})=\Delta \mu(\mathrm{H}, \mathrm{x}=0)$ are also material constants. In the sequel, the material characteristic $\Delta \mu_{\text {cut }}(\mathrm{H})$ is called maximal permeability drop due to cutting. This is 
understood as a change in the relative permeability of the material.

\subsubsection{The depth $\delta$ of the degraded zone is a lamination characteristic}

The depth $\boldsymbol{\delta}$ over which the material's permeability is degraded by the cutting depends on how the rupture stress diffuses across the material according to the laws of structural dynamics. It depends hence basically on the geometry of the lamination, in particular on the lamination thickness $\mathrm{d}$, as well as on structural elements such as the grain size. The punch-die clearance influences also the deformation, and consequently the apparition of dislocations and the degradation of the electrical steel performance. However, punchers know this phenomenon and work with optimal clearances for optimal cut edge shape. Such an optimal clearance was used in this study in order to make the results relevant for industrial punching applications.

\subsubsection{Spatial distribution of $\Delta \mu(\mathrm{H}, \mathrm{x})$}

Since the amplitude of the permeability drop $\Delta \mu_{\text {cut }}(\mathrm{H})$ is considered to be a material constant, the spatial distribution of $\Delta \mu(\mathrm{H}, \mathrm{x})$ can be expressed in terms of a field-independent spatial distribution function $\eta(x)$ as

$$
\Delta \mu(H, x)=\Delta \mu_{\text {cut }}(H) \eta(x) .
$$

Because the $\mathrm{H}$ field is homogenous over the sample, this spatial distribution is that of the $\mathrm{J}(\mathrm{x})$ field. It must have a localized support, i.e. $\eta .(x>\delta)=0$.

With this variable separation, one can calculate the average value of the magnetic permeability over the whole width $\mathrm{L}$ of the strip, assuming it is cut into $n_{p}$ pieces of equal width. If $L / N$, where $N$ is the number of cut-edges in the sample, is larger than the depth $\delta$ of the material degradation, the degraded zones associated with the different cuts do not overlap and are independent of each other. If $\delta>\mathrm{L} / \mathrm{N}$, the degraded zones of the cuts at both side of each piece overlap and it is assumed that the resultant distribution follows then

$$
\eta_{1+2}(x)=\max \left\langle\eta_{1}\left(x_{1}=x\right), \eta_{2}\left(x_{2}=L / N-x\right)\right\rangle .
$$

Taking the maximum is natural, as we deal with a quantity that is directly related with plastic strain. One can then readily show that the spatial average of the permeability $\mu(\mathrm{H}, \mathrm{x})$ over a sample with $\mathrm{N}$ cut edges evaluates as

$$
\bar{\mu}(H, N)=\mu(H, N=0)-\Delta \mu_{\text {cut }}(H) F(N),
$$

with

$$
F(N)=\frac{N}{L} \int_{0}^{\min (\delta, L / N)} \eta(x) d x
$$

\subsection{Resulting model for the cutting effect on $\mathbf{J}(\mathbf{H}, \mathbf{x})$}

Equation (3) is very useful to analyze the SST measurements. The spatial average permeability for various number of cut edges at the left-hand side of (3) is indeed directly known from the measurements (cfr. fig. 2), whereas the right-hand side contains two unknowns that one has to identify: the maximum permeability drop $\Delta \mu_{\text {cut }}(\mathrm{H})$, and the function $\mathrm{F}(\mathrm{N})$. The function $\mathrm{F}(\mathrm{N})$ itself depends on the number of cut edges $\mathrm{N}$ and the distribution function $\eta(x)$. It has the following properties:

- $\mathrm{F}(0)=0$

- $\mathrm{F}(\infty)=1$

- $\mathrm{F}(\mathrm{N})$ for $\mathrm{N}<\mathrm{L} / \delta$ is linear with slope $\frac{1}{L} \int_{0}^{\delta} \eta(x) d x$.

For the simple case of a parabolic distribution function

$$
\eta(x)=1-\frac{x}{\delta}-a \frac{x}{\delta}\left(1-\frac{x}{\delta}\right)
$$

the function $\mathrm{F}(\mathrm{N})$ is

$$
F(N)=\left\{\begin{aligned}
\frac{N \delta}{L}\left(\frac{1}{2}-\frac{a}{6}\right), & N \leq \frac{L}{\delta} \\
1-\frac{\mathrm{a}+1}{2}\left(\frac{\mathrm{L}}{\delta \mathrm{N}}\right)+\frac{a}{3}\left(\frac{\mathrm{L}}{\delta \mathrm{N}}\right)^{2}, & N \geq \frac{L}{\delta}
\end{aligned}\right.
$$


Additionally, $\eta(\mathrm{x})$ should also be determined on basis of considerations regarding

- the diffusion of stress and strain in plastic materials,

- the way plastic deformations due to successive cuttings actually add up,

- the intended FE implementation of the modified constitutive relationship.

The maximum permeability drop $\Delta \mu_{\text {cut }}(\mathrm{H})$, as discussed above, is a material constant and hence independent of $\mathrm{N}$. The left-hand side of the equation

$$
\Delta \mu_{\mathrm{cut}}(H)=\frac{\mu(H, N=0)-\bar{\mu}(H, N)}{F(N)} .
$$

should therefore not depend on $\mathrm{N}$, and one can use this fact to identify simultaneously $\Delta \mu_{\text {cut }}(\mathrm{H})$ and the values of $\mathrm{F}\left(\mathrm{N}_{\mathrm{k}}\right)$ for the number of cuts that have been measured.

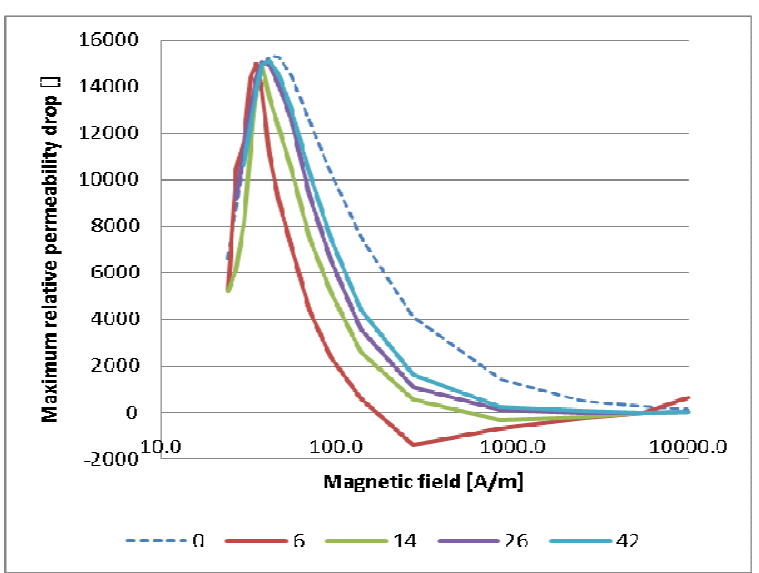

Figure 10: maximum relative permeability drop characteristics with different number of cutting edges, at $50 \mathrm{~Hz}$.

At $50 \mathrm{~Hz}$, the following results are obtained: fig. 10 shows the different expressions at the righthand side of equation (7), for $\mathrm{N}_{\mathrm{k}}=6,14,26$ and 42 , and the function $\mathrm{F}\left(\mathrm{N}_{\mathrm{k}}\right)$ identified so that the curves match as closely as possible with each other. Except for the sample with $\mathrm{N}=6$ cut-edges, a reasonable match is obtained, which defines the maximum permeability drop of the material $\Delta \mu_{\text {cut }}(\mathrm{H})$, see fig. 11 . It is observed that, at fields below $30-40 \mathrm{~A} / \mathrm{m}, \Delta \mu_{\text {cut }}(\mathrm{H})$ is nearly equal to the permeability of the uncut sample (represented with a dashed line). This means that the permeability of the cut material vanishes almost completely at very low fields. On the other hand, the permeability drop vanishes above $1000 \mathrm{~A} / \mathrm{m}$.

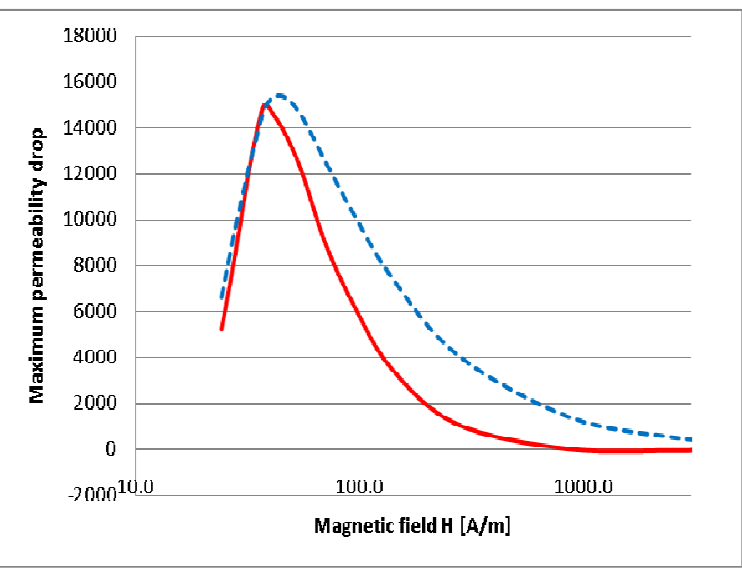

Figure 11: In red, the final identified maximum permeability drop $\Delta \mu_{\text {cut }}(\mathrm{H})$. As a comparison, the dashed line shows the relative permeability of the uncut material.

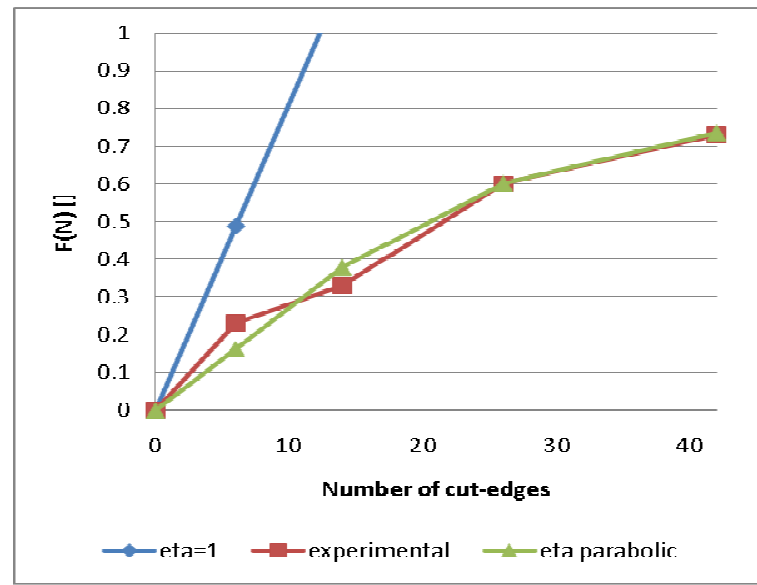

Figure 12: visualization of function $\mathrm{F}(\mathrm{N})$, both the one obtained from the experimental data, and the theoretical one fitted with $\eta$ a parabolic function, for the considered case at $50 \mathrm{~Hz}$. Also shown is the upper limit of $F(N)$ for $\eta=1$.

Fig. 12 shows the corresponding function $\mathrm{F}(\mathrm{N})$. The red curve (labeled "experimental") represents the values $F\left(\mathrm{~N}_{\mathrm{k}}\right)$ obtained from the identification process described above, whereas the green one (labeled "eta parabolic") represents the fitted theoretical $\mathrm{F}(\mathrm{N})$ function given in (6) corresponding to the parabolic distribution $\eta(x)$. The parameters describing the fitted distribution function $\eta(\mathrm{x})$ are found to be $\delta=6.5 \mathrm{~mm}$ and $\mathrm{a}=1.0$. The latter value shows that the distribution 
vanishes quadratically as $\mathrm{x}$ approaches $\delta$, as was shown experimentally by Nakata [3] who performed local magnetic measurements. Finally, the indicative blue curve (labeled "eta $=1$ ") represents worst case $\eta(\mathrm{x})=1,0<\mathrm{x}<\delta, \mathrm{F}(\mathrm{N})=\mathrm{N} \delta / \mathrm{L}$. This is the upper limit for all possible $\eta(\mathrm{x})$ functions.

The magnetization field distribution is reconstructed as

$$
J(H, x)=\mu_{0}\left(\mu_{r}(H, N=0)-\Delta \mu_{\text {cut }}(H) \eta(x)\right) H
$$

and Fig. 13 shows an example for a lamination cut on one side. As before, $\mathrm{x}=0$ corresponds with the cut edge.

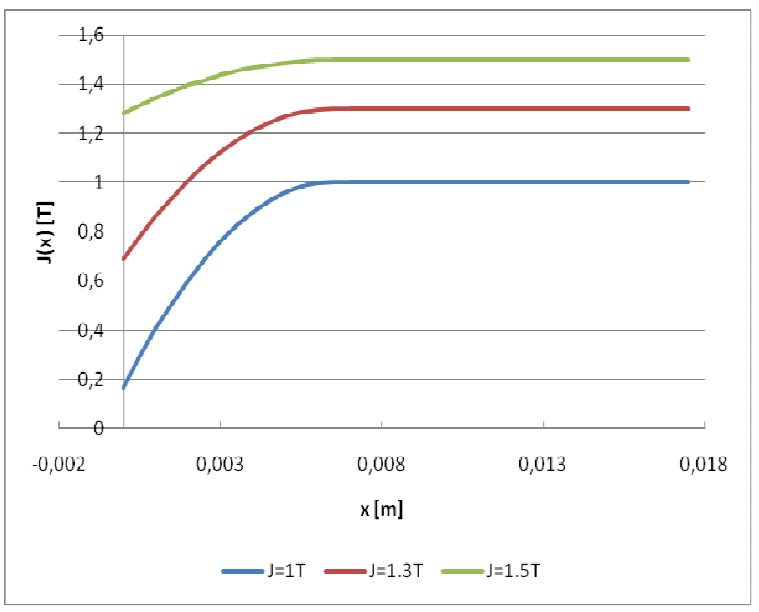

Figure 13: Distribution of $J(H, x)$ over the degraded zone for three different magnetic field strengths corresponding to bulk values of $\mathrm{J}=1 \mathrm{~T}, 1.3 \mathrm{~T}$ and $1.5 \mathrm{~T}$.

\section{Mathematic description of the effect of cutting on the losses $\mathbf{P}\left(\mathbf{J}_{\text {avg }}, \mathbf{x}\right)$}

In [1] we developed an improved loss model with the advantage to allow a closer fit to experimental measurements of iron losses, both in the application region of higher polarizations, as for higher frequencies.

$$
\begin{aligned}
\text { Ptot } & =a_{2} J(H)^{2} f \\
& +a_{1}\left(1+a_{3} J(H)^{a 4}\right) J(H)^{2} f^{2} \\
& +a_{5} J(H)^{1.5} f^{1.5}
\end{aligned}
$$

Besides providing adapted polarization curves at the edge zone for FEM field calculations, another target of this work is to implement the impact of cutting into the coefficients of the loss model [1] used in the post-processing. In (9), the term with $\mathrm{a}_{2}$ refers to the hysteresis losses, which are most prone to be affected by cutting, as discussed above in the experimental section showing the magnetization loops ( $\S 2.4$ ). Given the mathematical description as determined for the polarization and the permeability evolution in relation to the cutting impact, it is proposed to rewrite the equation as:

$$
\begin{aligned}
& \operatorname{Ptot}(x)=a_{2}(x) J(H, x)^{2} f \\
& \quad+a_{1}\left(1+a_{3} J(H, x)^{a 4}\right) J(H, x)^{2} f^{2} \\
& \quad+a_{5} J(H, x)^{1.5} f^{1.5}
\end{aligned}
$$

In this local loss description, the hysteresis coefficient $\mathrm{a}_{2}$ has become dependent on the distance from the cut edge. Furthermore we showed that near the cut edge $\mathbf{J}$ is deteriorated, whereas - for a given machine output - the average $\mathbf{J}$ over space needs to remain constant, hence $\mathbf{J}$ in the bulk will be larger. This implies $\mathbf{J}$ is not only dependent from $\mathrm{H}$, but also on $\mathrm{x}$.

\section{Conclusion}

The phenomenon of permeability decrease and iron loss increase due to cutting of the electrical steel laminations has been measured thoroughly in case of the Fully Processed M235-35A grade. A theoretical analysis has linked it to a decrease of the permeability at the vicinity of the cut-edge, and a mathematical description has been developed to allow calculating this effect in a quantitative way. At $50 \mathrm{~Hz}$, the impact of cutting is observed over a degraded region as deep as $6,5 \mathrm{~mm}$ from the cut-edge inwards. However, as the distribution is parabolic, the most significant material degradation occurs over the first $3 \mathrm{~mm}$ only. The degradation has also been shown to be more important at low fields:

- At 1T, the polarization drop at the cut-edge is $83 \%$, and this drop reduces to $24 \%$ at $3 \mathrm{~mm}$ from the cut-edge, compared to the bulk polarization value.

- At 1,5T, the polarization drop at the cutedge is $14 \%$, and this drop reduces to $4 \%$ at $3 \mathrm{~mm}$, compared to the bulk polarization value. 
Cutting increases iron losses in a twofold way:

- In a direct way, by increasing the hysteresis losses, and hence the total iron losses, at the vicinity of the cut edge.

- In an indirect way, by modifying the magnetization profile inside the lamination. Due to the permeability drop, the polarization obtained with a given excitation is significantly reduced at the cut edge. Hence, in order to have the same flux across the sample (i.e. the same average polarization), a higher polarization in the bulk of the lamination is required. This higher polarization induces higher total losses.

The mathematical description of the permeability loss defined in this paper, allows more correct finite element modeling of the field distribution in e-machines.

The next step is to introduce the permeability drop quantified in this work, and hence of the shape change of hysteresis loops, in the hysteresis coefficient of the advanced loss model described in our EVS24 paper [1]. In this way, both the field and the iron loss evaluation in e-machines will be done in a more accurate way. The result of this work is also that, via the impact of cutting on air gap flux, hence the need to adapt the magnetizing current, the possibility exists to make copper loss determination also more accurate.

\section{References}

[1] S. Jacobs, D. Hectors, F. Henrotte, M. Hafner, M. Herranz Gracia, K. Hameyer, P. Goes, "Magnetic material optimization for PMSM drives", World Electric Vehicle Journal Vol. 3, ISSN 2032-6653, 2009, AVERE.

[2] G. Bertotti, "General properties of power losses in soft ferromagnetic materials", IEEE Transactions on Magnetics, 24(1), pp. 621-630, January 1988.

[3] T. Nakata, M. Nakano, K. Kawahara, "Effects of Stress Due to cutting on Magnetic Characteristics on Silicon Steel", IEEE Translation Journal on Magnetic in Japan, Vol. 7, No. 6, June 1992.

[4] M. De Wulf, E. Hoferlin, L. Dupré, "Finite Element Modelling of Induction Machines under No-load Condition taking into account Manufacturing Processes", Proceedings ICEM 2004 (Cracow, Poland).

[5] M. De Wulf, "Aciers électriques non-orientés pour machines électriques et autres applications", Matériaux magnétiques en génie électrique, Tôme 1, Chapitre 2, edited by A. Kedous-Lebouc, Lavoisier, Paris (2006).

[6] A.J. Moses, N. Derebasi, G. Loisos, A. Schoppa, "Aspects of the cut-edge effect stress on the power loss and flux density distribution in electrical steel sheets", J. Magn. Mag. Mat, 215-216, June 2000, pp. 690-692.

[7] F. Ossart, E. Hug, O. Hubert, C. Buvat, R. Billardon, "Effect of punching on electrical steels: experimental and numerical coupled analysis", IEEE Trans. on Magn., Vol. 36, no. 5, 2000, p. 3137-3140.

[8] P. Baudouin, M. De Wulf, L. Kestens, Y. Houbaert, "The effect of guillotine clearance on the magnetic properties of electrical steels", J. of Magn. Magn. Mater., 256, 2003, p. 32-40.

[9] G. Crevecoeur, P. Sergeant, L. Dupré, L. Vandenbossche, R. Van de Walle, "Analysis of the local material degradation near cutting edges of electrical steel sheets", IEEE Trans. on Magn., vol. 44, no. 11, pp. 31733176, Nov 2008.

\section{Authors}

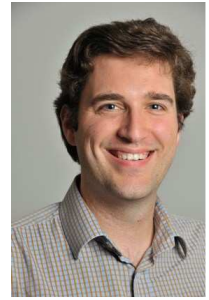

Dr. Ir. Lode Vandenbossche

ArcelorMittal Global R\&D Gent, J.F.Kennedylaan 3, B-9060 Zelzate, Belgium, +32 934513 84, lode. vandenbossche@arcelormittal.com.

Lode Vandenbossche graduated in electromechanical engineering at Gent University, Belgium, and then studied the link between magnetic properties and the microstructure of steels. This research about magnetic nondestructive evaluation of material degradation resulted in a $\mathrm{PhD}$ degree at Gent University. Currently he works at ArcelorMittal Global R\&D Gent, where he is performing research on electro-magnetic applications and electrical steel solutions.

Ir Sigrid Jacobs

ArcelorMittal Global R\&D, J.F. Kennedylaan 51, B-9042 Gent, Belgium, +32 $9342 \quad 23 \quad 12$, sigrid.jacobs@arcelormittal.com.

Sigrid Jacobs graduated in electrotechnical engineering at Ghent University and obtained an MBA at the Vlerick School for management, Belgium. After developing electrical steels at the metallurgy lab of the Ghent university, she joined the ArcelorMittal group and was involved

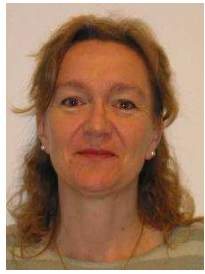
in the coupling project of a coupled pickling-tandem mill line, the development of electrical steels at different plants from the group and is involved in Global Customer Relations for the group's worldwide R\&D activities in Electrical Steels. 
Dr Ir Francois Henrotte.

IEM, RWTH Aachen University, Schinkelstrasse 4, 52056 Aachen, Germany, +49 2418097648, fh@iem.rwth-aachen.de.

François Henrotte graduated in engineering (Mechanics-Physics) and received the $\mathrm{PhD}$ in Applied Sciences at the University of Liège, Belgium. His main research interests are the numerical modelling of electromechanical systems and magnetic materials, the theoretical analysis of electromagnetic forces, differential geometry and applied mathematics. $\mathrm{He}$ is active in projects with industry, for development and optimisation of electrical motors.

Professor Kay Hameyer

IEM, RWTH-Aachen University, Schinkelstrasse 4, D-52056 Aachen, Germany, +49 $241809 \quad 7636$, kh@iem.rwth-aachen.de.

Kay Hameyer received his M.Sc. degree in electrical engineering from the University of Hannover and his Ph.D. degree from the Berlin University of Technology. He then worked with the Robert Bosch $\mathrm{GmbH}$ in Stuttgart as a Design Engineer for permanent magnet servo motors and board net components. He is currently the Director of the IEM and holder of the chair Electromagnetic Energy Conversion of the RWTH Aachen University, Germany. VDE, IEEE senior member.His research interests are numerical field computation, the design of electrical machines, and numerical optimisation strategies. 